\title{
Tangence
}

\section{L'intrigue policière et la Loi dans Agaguk d'Yves Thériault}

\section{Renald Bérubé}

Numéro 38, décembre 1992

Fiction policière et roman actuel

URI : https://id.erudit.org/iderudit/025736ar

DOI : https://doi.org/10.7202/025736ar

Aller au sommaire du numéro

Éditeur(s)

Tangence

ISSN

1189-4563 (imprimé)

1710-0305 (numérique)

Découvrir la revue

Citer cet article

Bérubé, R. (1992). L'intrigue policière et la Loi dans Agaguk d'Yves Thériault.

Tangence, (38), 33-41. https://doi.org/10.7202/025736ar d'utilisation que vous pouvez consulter en ligne.

https://apropos.erudit.org/fr/usagers/politique-dutilisation/ 


\section{L'intrigue policière et la Loi dans Agaguk d'Yves Thériault \\ Renald Bérubé}

Pour André Brochu

Quand on tire le pain de ses jours à jouer de ruse avec les bêtes, il serait incroyable que l'on ne devînt pas aussi rusé qu'elles. De la ruse à la déduction, qui est en somme prévoir le comportement des autres et en déduire des faits, il n'y a pas un long chemin à parcourir.

Yves Thériault, L'Étreinte de Vénus ${ }^{1}$

L'Inuk admet la force, la sait reconnaître, et se résigne à ce que le Blanc le domine. Puisque le Blanc est le plus fort.

Mais le jeu, c'est de vivre sa vie ancestrale, en défi constant aux Blancs.

[...] Mais déjouer un Blanc, policier de surcroît, pour agréable qu'en soit le sport, comporte des risques.

Yves Thériault, Agaguk ${ }^{2}$

[...] la nature énigmatique essentielle du roman policier, qui n'est pas seulement criminel, mais recourt toujours aux services d'un enquêteur: à mes

1 Yves Thériault, L'Étreinte de Vénus, Montréal, Éditions Québécor, 1981, p. 50

2 Yves Thériault, Agaguk, Montréal, Éditions de l'homme, 1963, p. 183. 
yeux aussi, toute fiction policière est fiction d'une enquête.

François Rivière, * La fiction policière ou le meurtre du roman. 3

Quand il entreprend la rédaction d'Agaguk, roman publié en 1958 et "roman esquimau "selon son générique, Yves Thériault a déjà derrière lui une solide pratique du genre policier. Selon une affirmation qu'il a répétée à l'occasion de nombreuses interviews, Thériault avait déjà écrit, sous divers pseudonymes, plusieurs "romans à dix cents" de la série *Guy Verchères, détective" notamment ${ }^{4}$. Plus tard, entre 1965 et 1968 , il sera l'auteur de huit romans pour la jeunesse publiés chez Lidec et mettant tous en scène *Volpek, l'agent secret canadien ; romans qui relèvent tout à la fois du policier, de l'espionnage et de la science-fiction, l'accent mis sur le policier ou la science-fiction, par exemple, pouvant varier selon les huit épisodes qui composent la séries. Mais Agaguk, en 1958, marque la première occurrence du genre policier dans le texte littéraire proprement dit de Thériault.

À quoi il faut tout de suite ajouter les trois ou quatre remarques suivantes. Tout d'abord que Thériault fera paraître en des lieux de publication divers, en 1958 et 1959, une série de contes policiers qui composeront plus tard l'essentiel du recueil intitulé L'étreinte de Vénus, recueil publié en 1981 et ayant précisément "contes policiers" comme générique; ensuite que Volpek, nom d'agent secret et donc pseudonyme, est ainsi nommé à partir de critères qui ne sont pas sans évoquer ceux qui président à la dénomination de Tayaout, premier enfant et premier fils du couple Agaguk-Iriook dans $A_{g a g u k^{6}}$; enfin, que ce roman,

3 François Rivière, - La fiction policière ou le meurtre du roman ", Europe, $\mathrm{n}^{*}$ 571-572 (numéro intitulé *La fiction policière.), novembre-décembre 1976 , p. 21.

4 Yves Thériault se raconte (entretiens avec André Carpentier), Montréal, VLB éditeur, 1985, p. 77 (note).

5 Les huit romans ont tous été publiés (en format poche) à Montréal chez Lidec: La montagne creuse (1965); Le secret de Mufjarti (1965); Les daupbins de Monsieur Yu (1966); Le château des Petits Hommes Verts (1966); Le dernier rayon (1966); La bête à 300 têtes (1967); Les pieuvres (1968); Les vampires de la rue Monsieur-le-Prince (1968).

6 J'ai abordé ce sujet des noms dans un article écrit en collaboration avec Françoise Daigle, L'attendu et l'inattendu: les aventures de Volpek, 
Agaguk, se situe, dans la production littéraire proprement dite de Thériault, à la suite notamment de La fille laide, premier roman de l'auteur publié en 1951, et d'Aaron (1954 et 1957, puisque ce roman a connu deux versions) qui précède immédiatement Agaguk. Or, La fille laide - question de meurtre commis par Fabien tout autant que question d'un enfant né de leur alliance mais que Fabien veut supprimer à l'encontre du désir de sa compagne Edith, la fille laide - contient des scènes qui se retrouveront à peu de choses près dans Agaguk Or, Aaron, à peu de choses près encore, se termine là où Agaguk commence: l'un et l'autre héros éponyme, à la fin d'Aaron dans le premier cas et au début d'Agaguk dans le second, se retrouvent dans une situation identique, celle de la rupture avec la tradition - juive dans le cas d'Aaron, inuit dans le cas d'Agaguk. Mais ni dans l'un ni dans l'autre roman, et malgré les parentés évidentes qu'ils entretiennent avec Agaguk; n'y a-t-il utilisation du genre policier.

Une quatrième et dernière remarque: Tayaout, fils d'Agaguk (1969) et Agoak (l'béritage d'Agaguk) (1975) forment, avec Agaguk dont ils prennent soin de rappeler le nom, une sorte de trilogie inuit. Dans Tayaout, le héros éponyme tuera son père, Agaguk, sans que pourtant cela ne mette en branle une intrigue policière; dans Agoak, le héros éponyme tuera deux Américains blancs qu'il surprend au moment où ils allaient violer Judith son épouse. Dès lors, le roman bascule et devient une impitoyable chasse policière - un roman noir au pays des neiges et des glaces. Dans la trilogie inuit en tout cas, il semble bien que l'intrigue de type policier ne doive se manifester que si des natives, pour employer l'expression d'un des deux Américains d'Agoak $^{7}$, s'en prennent à des Blancs.

Pour la bonne compréhension de ce qui va suivre, il vaut sans doute la peine de rappeler ici les événements qui, dans Agaguk, mettent en ceuvre l'intrigue de type policier. En nous rappelant par ailleurs que le résumé, selon Genette ${ }^{8}$, relève bien

l'agent secret canadien (premier parcours) , Études littéraires, vol. XXI, $\mathrm{n}^{\circ} 1$ (numéro intitulé *Yves Thériault, une écriture multiple.), printempsété 1988, p. 103-120.

7 Yves Thériault, Agoak (l'béritage d'Agaguk), Montréal, Stanké/Quinze, 1975, p. 142.

8 Gérard Genette, Palimpsestes (la littérature au second degré), Paris, Seuil, coll. «Pótique •, 1982, p. 282-283. 
des pratiques hypertextuelles; ce qui signifie aussi que tout résumé est mené selon l'optique choisie par celui ou celle qui résume et qu'il constitue dès lors, à sa manière, un métatexte ou texte critique. Pour tout dire, vous le savez, un résumé n'est jamais innocent, enquête policière obligeant, de la perspective critique de celui ou celle qui résume.

Tout au début d'Agaguk, donc, le héros titulaire, à dix-huit ans, quitte la tribu dont le chef est Ramook son père pour aller s'installer en un lieu situé davantage au *sud-est, vers le pays où vivaient, disait-on, des Sang-mêlés. (p. 11). Ce faisant, il rompt avec les mours ancestrales et prive la communauté d'un chasseur fort important pour la survie de celle-ci. Agaguk quitte la tribu parce qu'il se méfie des ruses de son père (p. 233-234); et parce que ce dernier ayant * pris une Montagnaise pour remplacer la femme morte, Agaguk considérait que la lignée était rompue" (p. 10). Le lieu de sa nouvelle vie reconnu et sommairement aménagé, Agaguk reviendra au village paternel chercher et ses biens et sa compagne, Iriook, libre absolument de toute attache parentale, ses père et mère étant décédés, qui a déjà accepté de vivre avec lui cette aventure inédite. Ainsi, Agaguk, dès son début et selon une situation fréquente dans la production de Thêriault, se présente selon une architecture binaire: il y a d'un côté le roman du village, de la tribu et de ses mœurs traditionnelles; de l'autre le roman d'amour Agaguk-Iriook qui sera aussi le lieu de la transformation (difficile) desdites mours, ce roman d'amour ayant de toute évidence les faveurs de l'instance narrative. D'une part la vie de la tribu, d'autre part celle du couple; surviendra bientôt un événement qui assurera la transition, la régulation narrative continue entre les deux lieux, entre les deux modes de vie opposés.

À l'invitation de Ghorok, sorcier en titre de Ramook, Agaguk, malgré les claires méfiances d'Iriook, retourne à son village natal pour échanger ses peaux. Un trafiquant blanc étant installé là depuis peu, a expliqué Ghorok, cela évitera à Agaguk le long voyage au comptoir d'échanges de la Compagnie de la Baie d'Hudson. Or, il se révèle que ce trafiquant blanc, Brown puisque tel est son nom et qui a un Montagnais comme acolyte, pratique un commerce hors-la-loi: il offre surtout de l'alcool en échange des peaux. Ce dont Agaguk ne veut pas, ce qui n'empêchera pas Brown de garder les peaux d'Agaguk. Lésé et vengeur, Agaguk reprendra son bien en faisant flamber Brown et la hutte qu'il habite, celle qui était la sienne quand il habitait le village de son père. 
Malgré les cadeaux de Ramook, le Montagnais dénoncera le meurtre de Brown à la Gendarmerie royale; si bien qu'un premier agent de la GRC, Henderson, se présentera bientôt au village. Où il sera accueilli par Ayallik, rival de Ramook à la chefferie de la tribu; Ayallik sera supprimé par Ramook et ses complices - et Henderson comprendra un peu tard qu'il ne peut rien contre la solidarité tribale. Il sera abattu de sang-froid et émasculé par Ramook.

La GRC ne saurait accepter la disparition d'un de ses agents qui enquêtait sur la mort d'un trafiquant blanc pourtant hors-la-loi; le village de Ramook verra alors arriver l'agent Scott et ses savants, sorciers de la race blanche. Tout cela se déroule à l'insu d'Agaguk que Ramook, coincé par Scott, dénoncera comme meurtrier de Brown et peut-être de Henderson; accompagné de Ramook et des siens, Scott et ses gens iront alors enquêter chez le couple. Mais Agaguk, défiguré à la suite de sa lutte contre le Grand Loup Blanc qui voulait s'en prendre à Tayaout, ne pourra être identifié de façon positive par Ramook; et Iriook, rusée et astucieuse, comprendra rapidement la situation dont pourtant elle n'avait nulle connaissance. Sa parole, alors que la parole est pourtant interdite à la femme inuit, répondra aux questions de Scott et sauvera Agaguk. S'étant plus tard compromis par vantardise, ayant succombé à la patience et à la ruse de Scott, Ramook sera dénoncé par les siens, de même que Ghorok - ils seront jugés chez et par les Blancs, et condamnés à la pendaison.

Et Agaguk, à qui Iriook a deux fois sauvé la vie - suite au combat contre le Grand Loup Blanc puis par ses réponses à Scott -, continuera selon un chemin plus ou moins tracé par cette dernière: il refusera la chefferie du village ${ }^{9}$ puis laissera vivre la fille à laquelle Iriook donne naissance - pour être aussitôt récompensé par la naissance d'un deuxième fils, jumeau de la fille. Raisonnements d'Iriook: contrairement à ce que croient les gens du village en lui offrant de devenir chef, d'avoir tué un Blanc et de n'avoir pas été puni par les Blancs ne fait pas d'Agaguk un héros. Et contre la vie enlevée au Blanc, il faut laisser la vie à la fille, à l'encontre des traditions inuit.

9 Qui avait êté offerte auparavant à des membres de la tribu, à Oonak par exemple, qui avait posé, entre autres conditions, qu'il y ait un : conseil des femmes" (p. 276); condition refusée (au grand dam de l'instance narrative), les Inuit y voyant * une façon des Blancs* (p. 277 ). 
La vie selon la tribu, la vie selon le couple; entre le roman de moeurs inuit et le roman d'amour du couple, l'intrigue policière. Car Agaguk a tué un Blanc dans le village de Ramook: ce qui met en cause tout à la fois et la tribu et le couple. Mais il n'y a pas ici que l'intervention d'une tierce instance, il y a aussi le fait que cette tierce instance est une instance étrangère. Dans Agaguk, "roman esquimau " selon le générique, la loi et la justice, ultimement, sont celles des Blancs. On comprendra dès lors que l'enquête policière, assurant la régulation diégétique entre les modes de vie opposés de la tribu et du couple et assurant aussi la fin définitive de Ramook et Ghorok déjà tous deux condamnés par le départ d'Agaguk au début du roman, on comprendra, donc, que cette enquête ait aussi à voir avec la quête de "la terre promise - - selon le titre du premier chapitre du roman, titre qui peut se souvenir d'Aaron, "roman juif " d'une certaine manière menée par Iriook et Agaguk. Comme on comprendra que sans présence humaine blanche dans l'Arctique, l'enquête de la GRC n'aurait jamais eu lieu. L'intrigue policière, dans Agaguk, est le signe manifeste de la cohabitation et de l'affrontement de deux systèmes de valeurs opposés; et elle signe le triomphe des valeurs blanches. Trois ou quatre pistes d'analyse (ou d'enquête) susceptibles de justifier et de valider ces hypothèses.

1. LE MEURTRE DE BROWN. Quand il se fait justice aux dépens de Brown, Agaguk se substitue, selon le mode inuit, tout à la fois au chef de la tribu, son père, et aux Blancs. En tant que chef du village en effet, Ramook ne devait pas laisser un Blanc exploiter honteusement les siens et mettre ainsi en péril la survie même de sa tribu; car à quoi pourra servir l'alcool, donné en échange des peaux, lorsque sévira l'hiver du Grand Nord? Que Brown soit accompagné d'un Montagnais, race honnie des Inuit, alors que Ramook vit avec une Montagnaise, ce qui a précipité le départ d'Agaguk de la tribu - puisque la lignée avait été rompue - mais qui n'a pas soulevé l'ire des autres, cela témoigne à la fois de l'emprise de Ramook sur les gens de sa tribu et du lien qui l'unit à l'exploiteur étranger Brown. En supprimant ce dernier, Agaguk, s'il agit pour des motifs personnels, souligne aussi que Ramook, au nom des seules valeurs des siens, ne devait pas laisser Brown commercer en sa tribu, ne devait pas assurer son pouvoir sur les siens par l'engourdissement alcoolisé de ceux-ci.

Mais en supprimant Brown, Agaguk applique aussi une loi blanche interdisant le trafic d'alcool avec les Inuit. Il connaît cette 
loi; et il l'applique avec plus de rigueur, sans doute, que les Blancs eux-mêmes, sa colère d'Inuk lésé par un étranger étant justement ce qu'elle est parce qu'il sait évaluer les préjudices que causent les échanges illicites de Brown. Faisant flamber avec ce dernier la hutte qu'il habitait autrefois, Agaguk biffe toute trace de son appartenance au village de son père, à sa propre ascendance biologicocollective, et applique la loi blanche mais selon le code inuit.

2. L'ENQUÊTE DE LA GRC. Si la seule présence de la Montagnaise auprès de Ramook avait déjà dénoncé celui-ci auprès d'Agaguk, le Montagnais accompagnant Brown dénoncera la tribu auprès de la GRC: Ramook le rusé n'apparaît ainsi que parce qu'il domine les siens - pour qui sait lire, il choisit mal ses alliances. (Car la ruse, si l'on se rappelle la première épigraphe, est pratique lectorale; l'instance qui lit doit savoir, rusée, enquêter, "déduire des faits ", etc.) L'enquête de la GRC sera mené en deux temps et se déroulera essentiellement à la tribu, à l'insu du couple, sauf à la fin. Comme si la seule collectivité avait des comptes à rendre. En un premier temps, l'agent Henderson viendra seul; sa maîtrise sera, littéralement, dévorée par celle du plus grand nombre. L'agent Scott arrivera en avion, sera entouré d'une équipe et vivra à l'écart de la tribu, dans l'avion justement; son langage mimera celui des Inuit (sorciers, magie), mais pour mieux démontrer la supériorité des sorciers/savants blancs. Ramook devra bientôt livrer un coupable: Agaguk, son fils, qui a tué Brown; et peut-être aussi, laisse-t-il entendre, Henderson. Sa pauvre ruse - faire porter chez Agaguk par Ghorok le fusil qui lui a servi à tuer Henderson - sera vite déjoué par son fils; et voulant montrer sa supériorité sur les sorciers blancs, Ramook révélera comment Henderson a été enterré. Dès lors, il a lui-même signé sa culpabilité et la tribu inuit le dénoncera à Scott et à sa atribu * blanche.

Dans Agaguk, l'enquête policière est affrontement des ruses - que l'instance lectorale peut d'autant mieux analyser que les meurtriers, les circonstances des meurtres, etc., lui sont connus, contrairement à ce qui se déroule dans le roman policier ainsi que défini par Roger Caillois et dans lequel le lecteur ou la lectrice doivent, comme l'enquêteur, tenter de reconstituer ce qui a déjà eu lieu ${ }^{10}$. Et le triomphe d'une ruse signe le triomphe d'une maîtrise qui a toutes les ressemblances de la loi. 
40

3. IRIOOK ET LA GRC. L'épisode est à juste titre célèbre et mérite qu'on s'y attarde afin de bien conclure. Après la dénonciation d'Agaguk par Ramook, Scott et ses gens de même que Ramook et les siens vont donc se rendre chez le couple - ce qui constituera la seule intrusion de l'enquête policière au lieu d'habitation d'Iriook et Agaguk. Ce dernier sait depuis bien peu la présence des agents à la tribu; depuis, de fait, qu'il a rapporté incognito chez Ramook le fusil qu'avait apporté chez lui Ghorok. Quant à Iriook, elle ne sait même pas qu'Agaguk a jadis tué Brown. Justement: situés l'un au début ou presque et l'autre à la fin ou presque du roman, ces deux épisodes s'appellent et se répondent. Si le meurtre de Brown enclenche l'enquête policière, les réponses d'Iriook à Scott mènent cette enquête vers son dénouement.

Si le couple ne sait rien ou presque de l'enquête qui se déroule au village depuis un moment déjà, Ramook ne sait rien des événements survenus chez le couple. Si bien que la défiguration d'Agaguk le laisse désemparé, rend impossible l'identification du fils par son père. Scott ayant brièvement expliqué les raisons de cette visite chez le couple, Iriook comprendra tout comme elle comprend tout depuis le début du roman, d'instinct ou d'origine, car elle vient du Dos de la Terre, là où demeurent les "derniers Esquimaux", selon le roman (p. 113). Iriook a d'abord sauvé Agaguk par ses gestes, suite au combat de celui-ci contre le Grand Loup Blanc; face à Scott, elle le sauvera par sa parole. Dans Agaguk, la parole d'Iriook sera la seule force inuit qui prévaudra face à la loi blanche. Contre toute tradition inuit qui laisse les palabres aux mâles, contre toute attente blanche s'agissant des Inuit.

Yves Lacroix, à la fin d'un article consacré à Agaguk, émettait l'hypothèse suivante: "la mère absente n'a pu désigner le père comme PORTEUR DE LA LOI "11. Hypothèse juste à un double titre: d'abord parce que Ramook, pourtant père et chef de la tribu à la fois, n'est porteur d'aucune loi sinon celle de son profit. Ensuite parce que le Blanc seul est véritable porteur de la loi et qu'Iriook, mère et épouse tout à la fois, peut seule parmi les Inuit, prévaloir face à la loi du Blanc. Prévaloir? Bien grand mot: car force est de constater que, sous la direction d'Iriook, Agaguk

11 Yves Lacroix, * Lecture d'Agaguk", Voix \& images, vol. V, n 2 (numéro dont le dossier est consacré à Yves Thériault), hiver 1980, p. 269. 
va vers des valeurs blanches (que sa direction d'origine au moment de quitter la tribu, le "sud-est * des "Sangs-mêlés", semblait déjà identifier). Dans le récit de la vie du couple, la narration, à la fin, se situe sous le signe d'Iriook comme son début était placé sous le signe d'Agaguk. De même que le meurtre de Brown défendait la survie des Inuit mais entérinait aussi la loi des Blancs, de même l'intervention d'Iriook face à Scott assurera la survie d'Agaguk, plus précisément du "nouvel * Agaguk et entérinera de fait les valeurs de la loi blanche. Phoquele-Père (p. 112), tué par Agaguk lors de la chasse miraculeuse, l'avait été aussi sur les indications données par Iriook à Agaguk (p. 127-135). Agaguk, fils d'Iriook.

La quatrième piste d'analyse (ou d'enquête), prendra la forme d'une conclusion. Qui pourrait se lire ou se dire ainsi: dans l'œuvre de Thériault, les forces en présence sont souvent catégoriquement opposées, noires ou blanches pourrait-t-on dire. Ce qui n'est certes pas sans attrait pour la fiction policière. Volpek saura faire ses choux gras de ce manichéisme. Et encore; car chez Thériault, une sorte de terrain neutre - ambivalent plutôt, "sangmêlé * en quelque sorte - traduit souvent la dualité des forces en présence. Dans Aaron par exemple, le mont Royal, lieu resté naturel alors même qu'il est situé en ville, regroupe tout à la fois les valeurs traditionnelles de Moishe et le désir de renouveau d'Aaron. Tel est, dans Agaguk, le rôle de l'intrigue médiane/ médiatrice policière: elle assure face à Scott le triomphe d'Iriook, Inuit du Dos ou du Sommet de la Terre, comme elle assure la prévalence, dorénavant, des valeurs de la très blanche GRC. L'une et l'autre, Iriook et la GRC, étant les vraies porteuses de la loi. Faut-il s'étonner, dès lors, que la femme d'Agoak se nomme Judith, prénom blanc (et biblique)? Et faut-il s'étonner qu'Agoak soit divisé en deux parties intitulées respectivement "les Esquimaux * et "les Inuit "? Car Agoak tirera ses propres conclusions du désir d'intégration aux valeurs blanches/esquimaudes et retournera férocement aux valeurs inuit de ses origines. Quitte à devoir subir une impitoyable chasse à l'homme, quitte à devoir survivre grâce à des ruses de bête traquée, loi de la jungle sur la toundra. 